\title{
Tagesschläfrigkeit bei obstruktiver Schlafapnoe (OSA) - pathogenetische Faktoren
}

\author{
Daytime Sleepiness in Patients with Obstructive Sleep Apnoea (OSA) - \\ Pathogenetic Factors
}

Autoren

Institute
U. Koehler ${ }^{1}$, S. Apelt' ${ }^{1}$ M. Augsten' ${ }^{1}$, F. Dette ${ }^{2}$, L. Jerrentrup ${ }^{1}$, J. Langanke' ${ }^{1}$, W. Cassel ${ }^{1}$

Klinik für Innere Medizin, SP Pneumologie, Intensiv- und Schlafmedizin (Direktor: Prof. Dr. C. Vogelmeier), Philipps-Universität Marburg

${ }^{2}$ Klinik für Anästhesie und Intensivtherapie (Direktor: Prof. Dr. H. Wulf), Philipps-Universität Marburg eingereicht 2.7.2010 akzeptiert nach Revision 20. 9.2010

\section{Bibliografie}

DOI http://dx.doi.org/ $10.1055 / \mathrm{s}-0030-1255838$

Online-Publikation: 26.11.2010

Pneumologie 2011; 65:

137-142 ๔ Georg Thieme

Verlag KG Stuttgart · New York ISSN 0934-8387

\section{Korrespondenzadresse}

Prof. Dr. Ulrich Koehler

Klinik für Innere Medizin

SP Pneumologie, Intensiv- und

Schlafmedizin

Universitätsklinikum Gießen

und Marburg GmbH

Standort Marburg

Baldingerstraße 1

35033 Marburg

koehleru@staff.uni-marburg.de

\section{Zusammenfassung \\ $\nabla$}

Tagesschläfrigkeit ist eines der führenden klinischen Symptome der obstruktiven Schlafapnoe (OSA). Diese ist mit einer eingeschränkten Lebensqualität sowie einer erhöhten Rate an Unfällen verbunden. Es leiden jedoch nicht alle Patienten mit OSA unter einer vermehrten Tagesschläfrigkeit. Die vorliegende Arbeit soll eine Übersicht über die relevanten Studien zum Thema OSA und Tagesschläfrigkeit vermitteln und Prädiktoren für Tagesschläfrigkeit herausarbeiten. Interessanterweise fand sich eine sehr große Heterogenität der untersuchten Parameter, sodass am ehesten von einer Multikausalität der Tagesschläfrigkeit auszugehen ist. Die Ausprägungen der Adipositas sowie der nächtlichen Atmungsstörungen scheinen pathogenetisch von besonderer Bedeutung zu sein. Die nichtinvasive nasale Beatmungstherapie führt bei Patienten mit OSA und Tagesschläfrigkeit in der Regel zu einer Verbesserung des klinischen Beschwerdebildes.

\section{Einleitung}

$\nabla$

Tagesschläfrigkeit bis hin zum imperativen Einschlafen sind führende klinische Symptome der obstruktiven Schlafapnoe (OSA). Entsprechend der ICSD-2 wird eine OSA dann diagnostiziert, wenn die Atmungsstörung durch keine andere Schlafstörung, anderweitige Erkrankung oder durch Medikamentengebrauch erklärbar ist [1]. Der Schweregrad sowie die Behandlungsnotwendigkeit der OSA wird anhand des Apnoe-Hypopnoe-Index (AHI), der klinischen Symptomatik (Tagesschläfrigkeit, Insomnieprobleme, fremdanamnestisch berichtete Atemstillstände) sowie der Begleitmorbidität (kardiovaskulär) beurteilt [1 3]. Bei einem obstruktiven AHI von $\geq 15 /$ h wird laut ICSD-2, ungeachtet der klinischen Symptomatik, von einer behandlungsbedürftigen OSA ausgegangen. Weniger stark ausgeprägte Schlaf-

\section{Abstract \\ $\nabla$}

Excessive daytime sleepiness (EDS) is one of the most frequent symptoms in patients with obstructive sleep apnoea (OSA). EDS can lead to substantial impairments in quality of life and is a major cause of fatal accidents. However, not all patients with OSA develop EDS. The aim of this paper is to review the current literature to identify factors having an impact on sleepiness in patients with OSA. Interestingly, a substantial heterogeneity of the results was found. Summarising these results, causes of EDS in patients with OSA are multifactorial. Severity of obesity and breathing disorders (apnoea/hypopnoea index) seem to be the most important predictors. Continuous positive airway pressure therapy significantly reduces sleepiness in patients with OSA.

apnoe (AHI $\geq 5$ bis $<15 / h$ ) sollte gemäß ICSD-2 nur dann behandelt werden, wenn sie von einer relevanten klinischen Symptomatik begleitet wird. Ab einem $\mathrm{AHI} \geq 15$ und $<30 / \mathrm{h}$ wird die OSA als mittel-, ab einem $\mathrm{AHI} \geq 30 / \mathrm{h}$ als schwergradig eingestuft. Faktoren, die das Auftreten von OSA begünstigen, sind in erster Linie Übergewicht, höheres Lebensalter, männliches Geschlecht und kraniofaziale Besonderheiten (Retrognathie).

Tagesschläfrigkeit verursacht erhebliche Leistungsdefizite und beeinträchtigt die kognitive und physische Leistungsfähigkeit. OSA-Patienten mit Tagesschläfrigkeit haben eine deutlich erhöhte Unfallwahrscheinlichkeit im Straßenverkehr. Während in einigen Untersuchungen eine positive Korrelation zwischen AHI und Tagesschläfrigkeit hergestellt werden kann, ist diese in anderen Untersuchungen nicht oder nur bedingt nachweisbar [4-8]. Die vorliegende Übersichtsarbeit 
macht es sich zur Aufgabe, den klinisch bedeutsamen Faktor „Tagesschläfrigkeit“ bei Patienten mit OSA näher zu beleuchten. Insbesondere interessiert der Sachverhalt, ob und welche Faktoren hinsichtlich der Ätiologie der Tagesschläfrigkeit bei Patienten mit OSA von klinischer Relevanz sind. Hierzu werden im Folgenden wichtige epidemiologische Studien und Schlaflaborstudien der jüngeren Zeit, die sich mit Schläfrigkeit bei Schlafapnoe, Schnarchen und Adipositas bei Erwachsenen und Kindern beschäftigen, zusammengefasst und bewertet.

\section{Definition der Tagesschläfrigkeit}

Tagesschläfrigkeit ist definiert durch eine Reduktion der zentralnervösen Aktivierung mit verminderter Wachheit, Daueraufmerksamkeit und Einschlafdrang [2,3,9]. Tagesschläfrigkeit verursacht Leistungsdefizite und beeinträchtigt die psychische und kognitive Leistungsfähigkeit sowie die allgemeine Lebensqualität. Sie ist unter anderem Folge nicht erholsamen Schlafs, weist einen Tagesgang mit Mittagstief auf und führt in monotonen Situationen oft mit kurzer Latenz zum Einschlafen. Tagesschläfrigkeit und die sich aus ihr ergebenden Einschränkungen am Arbeitsplatz, bei der Fahrtüchtigkeit sowie in sozialen Anforderungssituationen sind Folgen vieler Schlafstörungen, treten jedoch auch begleitend bei vielen anderen, vor allem chronischen Erkrankungen auf. Kennzeichen erhöhter Tageschläfrigkeit können Aufmerksamkeitsstörungen, Monotonieintoleranz, spontane und imperative Einschlafneigung bis hin zum Sekundenschlaf sein. Gegenüber der Tagesschläfrigkeit ist der Begriff der Müdigkeit abzugrenzen. Müdigkeit wird über das subjektive Gefühl von Erschöpfung und Mattigkeit beschrieben. Dies betrifft die Einschränkung sowohl motorischer als auch psychischer Funktionen. Patienten mit ausschließlich verstärkter Müdigkeit zeigen keine Monotonieintoleranz und keine Tagesschlafepisoden. In Situationen, in denen Schlaf möglich oder erwünscht ist, tritt dieser in der Regel nicht ein.

\section{Diagnostik der Tagesschläfrigkeit \\ $\nabla$}

In der Schlafmedizin steht der nicht erholsame Schlaf als Ursache der diagnostischen und therapeutischen Bemühungen im Rahmen der verstärkten Tagesschläfrigkeit im Vordergrund. Differenzialdiagnostisch gilt es neben Schlafstörungen körperliche Erkrankungen sowie situative Faktoren als potenzielle Ursachen vermehrter Tagesschläfrigkeit zu berücksichtigen. Tagesschläfrigkeit, vorliegend an mindestens drei Tagen pro Woche, wird in einer Häufigkeit von 4-20\% der untersuchten Stichproben beschrieben. Eine schwergradig ausgeprägte Tagesschläfrigkeit haben etwa 5\% der Untersuchten. Schlafmedizinische Beschwerden werden in erster Linie über Anamnese, Fragebögen zur Selbstbeurteilung sowie Interviews und Tagebücher ermittelt. Bei der Anamnese sollten Monotonieintoleranz, Einschlafneigung und Sekundenschlafepisoden in Art und Häufigkeit am Arbeitsplatz, im Straßenverkehr sowie unter anderen sozialen Bedingungen erfragt werden. Zur Beurteilung von Tagesschläfrigkeit hat sich die Epworth Sleepiness Scale (ESS) bewährt, sie dient der standardisierten Erfassung der erlebten habituellen Einschlafwahrscheinlichkeit in vorgegebenen Situationen [10,11]. Mit der ESS wird also nicht die momentane Schläfrigkeit erfragt, sondern die Einschlafneigung im Sinne einer überdauernden Disposition. Ein Testscore $\geq 11$ spricht für das Vorliegen pathologischer Tages- schläfrigkeit. Zu den apparativen diagnostischen Testverfahren zur Ermittlung der Ausprägung von Tagesschläfrigkeit zählen Multipler Schlaf-Latenz-Test (MSLT), Multipler Wachbleibe-Test (MWT), Vigilanztests (meist längerdauernde Reaktionszeittests wie der VigiMar-Test [12] und der Pupillografische Schläfrigkeitstest [9]. Während sowohl MSLT und MWT als auch Vigilanztests sowie die Pupillografie geeignet sind, Veränderungen unter Behandlung der Schlafapnoe zu objektivieren [9,13-15], zeigen sich eher schwache Korrelationen dieser Verfahren untereinander, mit Schlafvariablen und mit subjektiver Schläfrigkeit [13,16-19]. Während die Sensitivität dieser Verfahren für Behandlungseffekte auf eine relevante Assoziation mit der Erkrankung Schlafapnoe hinweist, spricht die geringe Interkorrelation dafür, dass mit den verschiedenen Testverfahren unterschiedliche Aspekte der Entität „Tagesschläfrigkeit“ erfasst werden [13]. Die recht geringe Korrelation der Verfahren zur Messung von Tagesschläfrigkeit zu Variablen aus der Polysomnografie wird oft recht einseitig diesen Testverfahren angelastet. Hier sollte stärker berücksichtigt werden, dass bisher sowohl nicht ausreichend klar ist, welche Parameter einer Schlafregistrierung (Tiefschlafanteil, Arousalindex, Apnoeindex, Schlafeffizienz) die Entmüdungsfunktion des Schlafs bestmöglich widerspiegelt [20], als auch bei Schlafvariablen eine recht große Nacht-zu-Nacht-Variabilität vorhanden ist [21]. Dies schränkt ihre Eignung als Validitätskriterium natürlich ein und man sollte daher nicht den Fehler machen, die mangelnde Beziehung von fragebogenbasierten oder apparativen Erfassungsmethoden der Tagesschläfrigkeit zu Schlafvariablen einseitig den Methoden zur Diagnostik der Schläfrigkeit anzulasten.

\section{Tagesschläfrigkeit bei Patienten mit OSA \\ $\nabla$}

Von Seiten des klinischen Aspekts erstaunt der Sachverhalt, dass nur ein vergleichsweise geringer Anteil von Patienten mit OSA unter einer ausgeprägten Tagesschläfrigkeit leidet. In der Wisconsin-Kohorte hatten beispielsweise nur 22,6\% der Frauen und $17,5 \%$ der Männer des Kollektivs der Patienten mit schlafbezogenen Atmungsstörungen ( $\mathrm{AHI} \geq 5 / \mathrm{h}$ ) eine vermehrte Tagesschläfrigkeit [22]. In der Penn State Kohorte beklagten 18\% der Patienten mit einem $A H I \geq 15 / h$ eine vermehrte Tagesschläfrigkeit, in der Sleep Heart Health Studie waren es $46 \%$ der Patienten mit einem AHI $\geq 15 / h[5,23]$.

Hat man täglich mit diesen Patienten zu tun, so imponiert der Eindruck, dass mehr als 80\% Befindlichkeitsstörungen am Tage, insbesondere eine vermehrte Tagesschläfrigkeit, beklagen. Dies mag damit zusammenhängen, dass sich primär ein hochgradig vorselektioniertes Patientenklientel im schlafmedizinischen Zentrum vorstellt, in dem sich eher schläfrige Patienten, die ja dadurch Leidensdruck erleben, wiederfinden als in epidemiologischen Studien, wo der Untersucher auf den nicht klagenden Proband zugeht und ihn untersucht. Im Folgenden sollen die Ergebnisse einiger Studien ausführlicher dargestellt und diskutiert werden. Im Speziellen ist die Frage von Interesse, ob es prädiktive Faktoren für das Vorliegen einer verstärkten Tagesschläfrigkeit bei Patienten mit OSA und Patienten mit Adipositas ohne OSA gibt. Auch auf die Frage der Reduzierung von Tagesschläfrigkeit unter nichtinvasiver Beatmung soll eingegangen werden.

Roure et al. [6] verglichen 1649 Patienten mit OSA und Tagesschläfrigkeit (ESS-Score; MW 15 \pm 3 ) mit 1233 OSA-Patienten

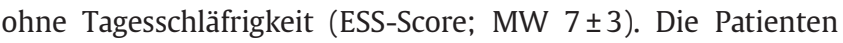
mit OSA und Tagesschläfrigkeit hatten, im Vergleich zu Patienten 
mit OSA ohne Tagesschläfrigkeit, einen leichtgradig höheren AHI $(36 \pm 27$ vs $33 \pm 25 / h)$ und Arousal-Index ( $37 \pm 31$ vs $33 \pm 23 / h$ ), eine niedrigere minimale Sauerstoffsättigung sowie eine bessere Schlafeffizienz und geringere Einschlaflatenz. Die Patienten mit OSA und Tagesschläfrigkeit waren im Vergleich etwas jünger ( $51 \pm 12$ vs $54 \pm 13 \mathrm{~J})$.

Gottlieb et. al [7] konnten bei 1824 Patienten der Sleep Heart Health Study, davon 886 Männer und 938 Frauen (mittleres Alter 65 Jahre), eine Abhängigkeit der Tagesschläfrigkeit von der Ausprägung der Atmungsstörung - hier Höhe des Respiratory-Disturbance-Index (RDI) - RDI nachweisen. Entsprechend der Kategorisierung des RDI in 4 Gruppen ( $<5 / \mathrm{h}, 5$ bis $<15 / \mathrm{h}, 15$ bis $<30 /$ $\mathrm{h}, \geq 30 / \mathrm{h}$ ) fand sich ein Anstieg des ESS-Scores von 7,2 (RDI $<5 / \mathrm{h})$ auf 9,3 (RDI $\geq 30 / h$ ). Eine Abhängigkeit der Tagesschläfrigkeit von Alter, Geschlecht oder BMI war nicht nachweisbar. Der Anteil der Patienten mit erhöhter Tagesschläfrigkeit (ESS-Score $\geq 11$ ) betrug $21 \%$ bei Patienten mit einem RDI $<5 / h$ und $35 \%$ bei Patienten mit einem RDI $\geq 30 / h$.

Bixler u. Mitarb. [5] untersuchten die Einflussnahme verschiedenster Faktoren auf die Tagesschläfrigkeit bei 16583 Frauen und Männer eines Bevölkerungsquerschnitts (Penn State Cohorte) im Alter von 20 bis 100 Jahren (MW 46,5 J).

Gesteigerte Tagesschläfrigkeit wurde in dieser Untersuchung leider nicht mit der ESS erfasst, sondern wie folgt definiert:

- „Fühlen Sie sich während der meisten Zeit des Tages schläfrig oder dösig, schaffen es aber, wach zu bleiben?" (Do you feel drowsy or sleepy most oft the day but manage to stay awake?)

- „Haben Sie während des Tages unwiderstehlich Schlafatta-

cken?" (Do you have any irrestistible sleep attacks during the day?)

Wenn bei einer der beiden Fragen als Ausprägung „moderat“ oder „schwer“ angegeben wurde, galt dies als Beleg für gesteigerte Tagesschläfrigkeit.

Der BMI lag im Mittel bei 26,3 kg/m² mit einem Bereich von 18 bis $69 \mathrm{~kg} / \mathrm{m}^{2}$. Die Prävalenz erhöhter Tagesschläfrigkeit betrug 8,7\% im Gesamtkollektiv. Die Diagnose einer Depression war mit 13,3\% vertreten, die eines Diabetes mellitus mit 13,0\%. Bei 1741 Patienten der Kohorte wurde zudem eine einmalige Untersuchung im Schlaflabor durchgeführt. Ein AHI >15/h konnte bei 4,4\% der Patienten gefunden werden. Den Ergebnissen der Untersuchungen zufolge war die erhöhte Tagesschläfrigkeit am engsten mit den Diagnosen Depression, Adipositas und Diabetes mellitus verknüpft. Ein signifikanter Zusammenhang zwischen obstruktiver Schlafapnoe und Tagesschläfrigkeit war der multiplen Regressionsanalyse zufolge nicht nachweisbar. Hier muss allerdings beachtet werden, dass ein eventueller gemeinsamer Einfluss von Adipositas und Atmungsstörungen methodenbedingt bei einer Regressionsanalyse, hier z. B. der Adipositas, zugeschrieben worden sein könnte. Die Häufigkeit erhöhter Tagesschläfrigkeit betrug bei Patienten mit einem AHI > 15/h 18,3\%, bei den Patienten ohne schlafbezogene Atmungsstörungen 10,7\%.

Oksenberg et al. [4] untersuchten 644 Patienten mit ausgeprägter OSA (AHI $\geq 30 / h$ ) polysomnografisch. ESS-Daten konnten bei 569 Patienten (88,3\%) erhoben werden. 327 der 569 Patienten (57,5\%) hatten einen ESS-Score $\geq 11$. Die Autoren fanden bei den Patienten mit ESS $\geq 11$ im Vergleich zu denen mit ESS < 11 einen signifikant erhöhten AHI (67,2 $\pm 21,6 / \mathrm{h}$ vs. 57,1 $\pm 18,3 / \mathrm{h})$ und ArousalIndex (65,5 $\pm 21,3$ vs. 58,0 $\pm 20,5 / h)$. Der BMI betrug bei den Patienten mit ESS $\geq 1134,3 \pm 5,5 \mathrm{~kg}$, bei den Patienten mit ESS $<11$ $33,0 \pm 5,2 \mathrm{~kg}$. Die minimale Blutgassättigung in REM- und NREMSchlaf war bei den OSA-Patienten mit ESS $\geq 11$ niedriger als beim Vergleichskollektiv $(67,5 \pm 16,1 \%$ vs. $74,3 \pm 13,4 \%$ und 77,1 $\pm 10,1 \%$ vs $80,5 \pm 7,6 \%$ ). Die Schlaflatenz war bei den Patienten mit ESS >11 geringer, die Schlafeffizienz nicht unterschiedlich. Chen u. Mitarb. [8] untersuchten 1035 Patienten (Alter $45 \pm 15$ Jahre; BMI 26,6 $\pm 4,3 \mathrm{~kg} / \mathrm{m}^{2}$ ) mit Schnarchen mittels Polysomnografie. Begleitend wurde die Schläfrigkeit anhand der ESS objektiviert. 24,1\% der Patienten hatten einen $\mathrm{AHI}<5 / \mathrm{h}, 21,7 \%$ einen AHI von $5-20 / h, 16,5 \%$ einen $A H I \geq 20-40 /$ h sowie $37,7 \%$ einen schwergradigen Befund mit einem AHI $>40 /$ h. Entsprechend der multiplen Regressionsanalyse zeigte sich ein signifikanter $\mathrm{Zu}$ sammenhang des ESS-Scores mit dem AHI, dem BMI sowie dem Entsättigungs-Index.

Dixon et al. [24] untersuchten 331 von 1055 extrem adipösen Patienten (BMI $\geq 35 \mathrm{~kg} / \mathrm{m}^{2}$ ) vor Magenbanding im Schlaflabor polysomnografisch. Sie fanden keine signifikante Korrelation zwischen ESS und den Parametern der Polysomnografie, insbesondere keine zum AHI. Der ESS-Score wurde mit 9,9 \pm 5,3 bestimmt, der AHI war mit 15,1/h (Range 6,0 bis 35/h) vergleichsweise niedrig. Der mittlere ESS-Score wurde bei Patienten mit einem AHI zwischen 0 und 15 mit 9,9 5,0 angegeben, der bei Patienten mit $A H I \geq 15 /$ h war mit 9,97 $\pm 5,1$ praktisch identisch. Hier ist anzumerken, dass durch die Auswahl einer Extremgruppe (BMI $\geq 35 \mathrm{~kg} / \mathrm{m}^{2}$ ) Zusammenhänge, die in einer unausgewählten Population vorhanden sind, maskiert werden können.

Von den Teilnehmern der Sleep Heart Health Study (6440) hatten 1149 einen AHI $\geq 15 / h$ [23]. Bei 1115 der 1149 Personen lag ein ESS-Scoring vor. 510 Teilnehmer $(45,7 \%)$ hatten einen ESS-Score $\geq 11,605$ einen Score < 11. Die beiden Subkollektive unterschieden sich vor allem im Hinblick auf Komorbidität, Schlafdauer, insomnische Beschwerden und periodische Beinbewegungen. Bei den Komorbiditäten waren die COPD und das Asthma bronchiale bei den tagesschläfrigen Patienten signifikant häufiger. Der mittlere AHI war bei den Probanden mit ESS $\geq 11$ mit 31,6/h leichtgradig höher als beim Vergleichskollektiv (28,6/h), ebenso zeigten Patienten mit Tagesschläfrigkeit etwas niedrigere Sauerstoffsättigungswerte. Ein Zusammenhang zwischen Tagesschläfrigkeit und Schlaffragmentation war nicht nachweisbar.

Mediano et al. [25] verglichen 23 männliche Patienten mit OSA (AHI $62 \pm 18 / \mathrm{h}$ ) und verstärkter Tagesschläfrigkeit (ESS $17 \pm 3$; MSLT-Score $4 \mathrm{~min}$ ) mit 17 bezüglich Geschlecht, Alter und BMI gepaarten Patienten (AHI $60 \pm 20 / h$ ) ohne Tagesschläfrigkeit (ESS $5 \pm 2$; MSLT-Score $16 \mathrm{~min}$ ). Die Patienten mit OSA und verstärkter Tagesschläfrigkeit hatten eine erhöhte Schlafeffizienz, eine kürzere Einschlaflatenz sowie signifikant niedrigere Werte von minimaler ( $69 \pm 12$ vs. $79 \pm 8 \%$ ) und mittlerer ( $87 \pm 6$ vs. $90 \pm 5 \%$ ) Sauerstoffsättigung. Die Schlafstadienverteilung sowie der Arousalindex unterschieden sich nicht signifikant.

Tsaoussoglou et al. [26] untersuchten 150 Kinder im Alter von 5 bis 17 Jahren polysomnografisch im Hinblick auf einen Zusammenhang zwischen Adipositas, SBAS, Tagesschläfrigkeit und Inflammationsparametern. Die Häufigkeit der Tagesschläfrigkeit war am höchsten in der Gruppe der Kinder mit Übergewicht und OSA (70\%), deutlich niedriger in der Kontrollgruppe mit Adipositas ohne OSA, am niedrigsten in der Kontrollgruppe ohne Übergewicht und ohne OSA.

Die polysomnografische Diagnostik bei 50 adipösen schnarchenden Kindern und 50 alters- und geschlechtsgepaarten nichtübergewichtigen Kindern ergab einen vergleichbaren AHI mit 10,9 $\pm 1,5$ vs $12,0 \pm 1,7 / \mathrm{h}$, die mittlere Schlaflatenz war jedoch in der Gruppe der adipösen Kinder signifikant kürzer (12,9 $\pm 0,9$ vs $17,9 \pm 0,7)[27]$.

Unter dem Aspekt der hohen Koinzidenz von Adipositas und OSA liegt die Frage nahe, ob die Adipositas per se zu einer klinisch re- 
levanten Tagesschläfrigkeit führt. Vgontzas u. Mitarb. [28] konnten bei immerhin 42 von 73 adipösen Patienten (57\%) mit polysomnografisch ausgeschlossener OSA eine vermehrte Tagesschläfrigkeit (im Mittel moderat) nachweisen. Lediglich einer von 45 Probanden des Kontrollkollektivs (altersgepaart, keine Adipositas) hatte eine milde Tagesschläfrigkeit. Tagesschläfrigkeit wurde in dieser Untersuchung leider nicht per ESS erfragt. Es wird lediglich berichtet, dass Tagesschläfrigkeit subjektiv mittels einer 3-Punkt-Rating-Skala (mild, moderat, schwer) in einem Schlaffragebogen erhoben wurde.

Die Arbeitsgruppen von Roure und Vgontzas [6,29-31] formulieren im Hinblick auf das Vorliegen erhöhter Tagesschläfrigkeit bei Adipositas und OSA einen „metabolisch-inflammatorischen Ansatz“. Die Autoren fanden sowohl bei Patienten mit ausschließlicher Adipositas bzw. OSA erhöhte TNF-alpha- sowie IL6-Werte. Bei der Koexistenz von Adipositas und OSA waren beide Parameter, im Sinne einer Potenzierung der inflammatorischen Antwort, am stärksten erhöht. Bei Alter, Adipositas, Diabetes mellitus, Insulinresistenz, Bewegung und Depression fanden die Autoren einen relevanten Zusammenhang mit der Ausprägung der Tagesschläfrigkeit. De la Pena Bravo u. Mitarb. [32] konnten bei Patienten mit OSA ebenfalls erhöhte Inflammationsparameter nachweisen, sie fanden jedoch keinen relevanten Unterschied von TNF-alpha, IL-6 und ICAM-1 zwischen OSA-Patienten mit und ohne Tagesschläfrigkeit.

In Tab. 1 sind Studien, Studiensetting, Erfassungsmethode der Tagesschläfrigkeit sowie die Einflussgrößen auf die Tagesschläfrigkeit im Überblick dargestellt.

Unter der nasalen CPAP-Therapie, die als Standardbehandlung der obstruktiven Schlafapnoe anzusehen ist, können die klinischen Symptome sowie das kardiovaskuläre Risiko reduziert werden. Im Hinblick auf die Tagesschläfrigkeit ergibt sich ein weitgehend eindeutiges Bild dahingehend, dass vor allem Patienten mit schwergradiger OSA und ausgeprägter Tagesschläfrigkeit von einer CPAP-Therapie profitieren [33,34]. Den Ergebnissen der Metaanalyse von Patel u. Mitarb. [34] zufolge, fand sich der ESS-Score bei 706 Patienten mit OSA unter CPAP im Mittel um 2,94 Punkte reduziert. Patienten mit schwergradiger OSA (AHI $>30 / h$ ) und gleichzeitig bestehender Tagesschläfrigkeit (ESS $\geq 11$ ) hatten eine deutlich ausgeprägtere Reduktion des ESSScores. Die sogenannte residuale Tagesschläfrigkeit [35], also unter nCPAP-Behandlung weiterbestehende Tagesschläfrigkeit, ist nicht Gegenstand dieses Literaturüberblicks, wir erlauben uns aber die Anmerkung, dass es sich hier möglicherweise um einen Terminus handelt, der eher als Sammelbegriff für unzureichende nCPAP-Einstellung, mangelnde Compliance, Übersehen anderer schläfrigkeitsverursachender Faktoren anzusehen ist als um die Bezeichnung eines definierten Krankheitsbildes.

\section{Bewertung und Ausblick}

$\nabla$

Als ursächlich für die vermehrte Tagesschläfrigkeit bei OSA-Patienten wurden bislang die „zerstörte Schlafstruktur“ sowie der „intermittierend auftretende Sauerstoffmangel“ angesehen. Geht man von dem pathogenetischen Konstrukt der „Entmüdungsfunktion des Tiefschlafs“ aus, so erscheint einleuchtend, dass eine fragmentierte Schlafstruktur bei reduziertem NREM III- und IV-Anteil eine verstärkte Tagesschläfrigkeit bedingen kann. Tiefschlaf gilt als engstes Korrelat der Entmüdungsfunktion des Schlafes und nimmt einen relativen Anteil von 15-20\% der Gesamtschlafzeit ein [1 -3]. In der hier vorgestellten Literaturre- cherche wurde in 4 von 7 Arbeiten kein klarer Zusammenhang von Tagesschläfrigkeit und Tiefschlafanteil in der Polysomnografie gefunden; bei 2 Arbeiten ging niedriger, bei einer aber auch erhöhter Tiefschlafanteil mit gesteigerter Tagesschläfrigkeit einher.

Unumstritten ist der Sachverhalt, dass die habituelle Schlafdauer eines Menschen genetisch determiniert ist und einer interindividuellen Variation unterliegt. So existiert eine Schwankungsbreite in der Schlafdauer der Normalbevölkerung von fünf bis neun Stunden mit einem Mittelwert von etwa 7,5 Stunden. Kurzschläfer mit einer durchschnittlichen Schlafdauer von 4 bis 5 Stunden weisen in der Regel im Vergleich zu Normalschläfern weniger NREM-Schlaf I und II auf, haben jedoch hinsichtlich des Tiefund REM-Schlafs interessanterweise keine quantitativen Defizite. Aus diesem Sachverhalt hat sich die Auffassung abgeleitet, dass eine definierte Menge an Tiefschlaf für die Entmüdungsfunktion des Schlafes von elementarer Bedeutung ist. In diesem Zusammenhang ist interessant, dass in nur 3 der hier vorgestellten 13 Studien der Einfluss der Schlafdauer auf die Tagesschläfrigkeit berücksichtigt wurde. Hier wurde, der Alltagserfahrung entsprechend, zweimal zunehmende Tagesschläfrigkeit bei kürzerer Schlafdauer gefunden. In einer Untersuchung ergab sich kein $\mathrm{Zu}-$ sammenhang (vgl. @ Tab. 1).

Viele Studien belegen zwar einen Zusammenhang zwischen Tagesschläfrigkeit und der Ausprägung der SBAS bzw. dem AHI, dieser ist aber nicht so eng, wie bislang gemutmaßt. 10 der 13 hier vorgestellten Studien berücksichtigen diesen Faktor. Viermal wird kein bedeutsamer Zusammenhang gefunden, in 6 Studien konnte eine zunehmende Tagesschläfrigkeit bei ausgeprägteren Atmungsstörungen belegt werden. Ähnlich eng stellt sich die Beziehung zwischen nächtlicher Sauerstoffsättigung und Tagesschläfrigkeit dar: Viermal wurde kein deutlicher Zusammenhang beobachtet, fünfmal geht gesteigerte Tagesschläfrigkeit mit niedrigerer Sauerstoffsättigung einher. Die Auffassung einer „Multikausalität“ der Tagesschläfrigkeit wird unter anderem auch dadurch bekräftigt, dass es zum einen Patienten mit ausgeprägter Atmungsstörung, aber keiner relevanten Tagesschläfrigkeit, zum anderen Patienten mit geringgradig erhöhtem AHI bei exzessiver Tagesschläfrigkeit gibt.

Tagesschläfrigkeit bei OSA ist am ehesten als ein multifaktoriell bedingtes Symptom aufzufassen. Pathophysiologisch bedeutsam erscheinen Adipositas und Ausprägung der schlafbezogenen Atmungsstörung. So ist augenscheinlich, dass vor allem adipöse Patienten mit hohem AHI unter einer exzessiven Tagesschläfrigkeit leiden. Die „metabolisch-inflammatorische Hypothese“ der Tagesschläfrigkeit ist zweifellos interessant, bedarf jedoch weiterer Grundlagenforschung. Faktoren, die die Lebensqualität abbilden, sollten stärker berücksichtigt werden, beispielsweise der psychologisch-psychiatrische Status, Insomnien und Komorbiditäten. Interessant ist der Sachverhalt, dass in zwei Studien, die das Morbiditätsspektrum der Patienten mit OSA berücksichtigen, respiratorische Erkrankungen wie COPD und Asthma deutlich häufiger bei Patienten mit vermehrter Tagesschläfrigkeit zu finden waren $[23,36]$. Bei älteren Patienten sind chronische Begleiterkrankungen sowie Medikamentennebenwirkungen, die auf die zentralnervöse Aktivierung Einfluss nehmen, abzuklären. Ebenso stellt Schlafmangel möglicherweise ein unterschätztes Problem dar. Problematisch ist bei den bislang durchgeführten Studien, dass die vom Patienten subjektiv beurteilte Tagesschläfrigkeit mit unterschiedlichen Messmethoden abgebildet wird. In einigen Studien kommen nicht einheitlich strukturierte Fragebögen zum Einsatz, in anderen die Epworth Sleepiness Scale. Die derzeit zur 


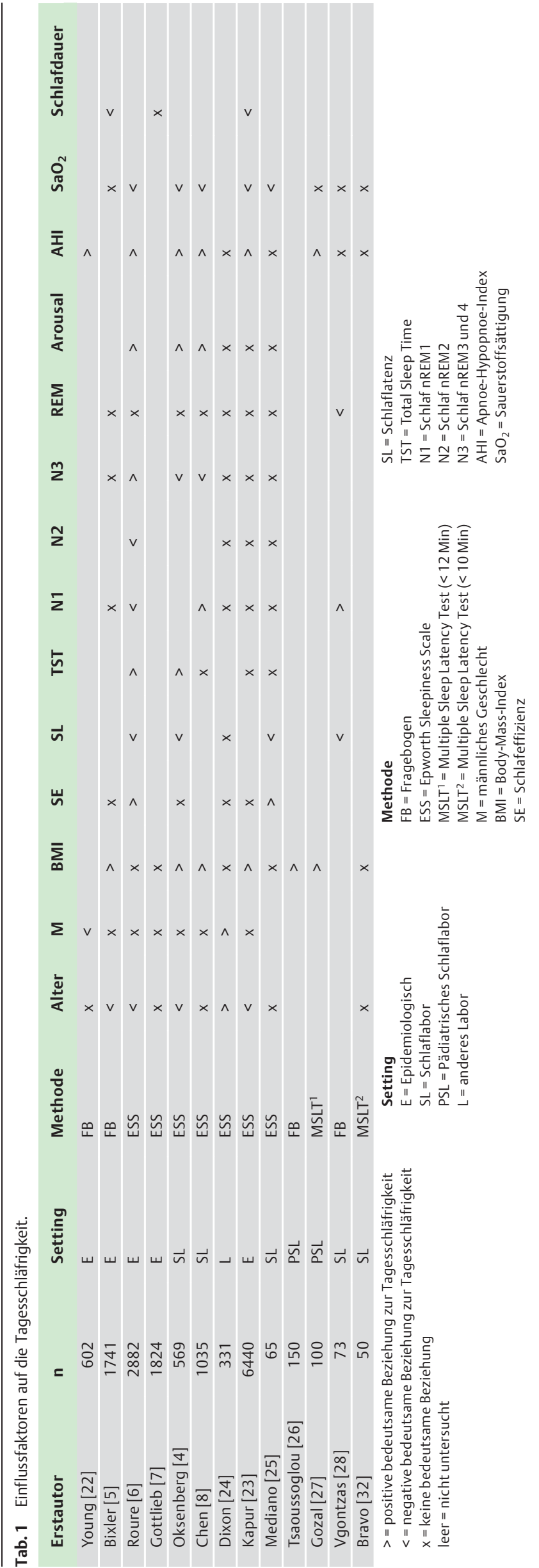

Verfügung stehenden apparativen Testverfahren zur objektiven Beurteilung von Tagesschläfrigkeit und Vigilanz haben in der Schlafmedizin eine große Akzeptanz, korrelieren aber nicht besonders eng mit Schlafparametern [13], mit subjektiver Schläfrigkeit $[17,18]$, erlebter Beeinträchtigung durch die Erkrankung [17]. Auch untereinander korrespondieren sie nicht besonders eng $[13,16]$. Objektive Messverfahren zur Beurteilung von müdigkeits- und schläfrigkeitsbezogenen Einschränkungen bilden somit wohl verschiedene Facetten des Konstruktes Tagesschläfrigkeit $\mathrm{ab}$ und sollten demzufolge sinnvoll kombiniert werden: führt man z.B. den zur Differenzialdiagnostik der Tagesschläfrigkeit empfehlenswerten MSLT durch, der auch Einschlaffähigkeit misst, sollte dieser z.B. mit einem Vigilanztestverfahren kombiniert werden, das Reaktionsbereitschaft unter monotonen Bedingungen und somit die Wachbleibefähigkeit erfasst. Zusätzlich ist es sinnvoll, die erlebte Schläfrigkeit in standardisierter Weise z.B. mit der ESS zu erfassen.

Als Limitation dieser Literaturübersicht ist deren heuristisch-deskriptiver Charakter zu sehen, in dessen Rahmen Arbeiten ausgewählt wurden, die nach Ansicht der Autoren die Problematik des Zusammenhangs zwischen Schlafapnoe und Tagesschläfrigkeit sehr gut illustrieren.

\section{Fazit}

- Tagesschläfrigkeit bei OSA ist am ehesten multifaktoriell bedingt. Bedeutsame Faktoren dürften sein: Höhergradige Adipositas und AHI.

- Schlafstrukturveränderungen sowie die Anzahl der Arousal scheinen weniger bedeutsam als bislang angenommen.

- Einflüsse von Begleitmorbidität und Medikamenten müssen bei zukünftigen Studien stärker berücksichtigt werden.

- Von der nichtinvasiven Beatmung profitieren im Hinblick auf die Tagesschläfrigkeit in erster Linie Patienten mit ausgeprägter OSA.

- Subjektive und objektive Verfahren zur validen Beurteilung von Tagesschläfrigkeit sollten kombiniert werden.

\section{Interessenkonflikt}

Die Autoren geben an, dass kein Interessenkonflikt besteht.

\section{Literatur}

1 American Academy of Sleep Medicine. International classification of sleep disorders. Diagnostic and coding manual; 2nd ed. Westchester, Illinois: American Academy of Sleep Medicine; 2005

2 Mayer G, Fietze I, Fischer J et al. S3-Leitlinie Nicht-erholsamer Schlaf/ Schlafstörungen. Somnologie 2009; 13: 4-160, Supplement 1

3 Peter H, Penzel T, Peter JH. Enzyklopädie der Schlafmedizin. Heidelberg: Springer; 2007

4 Oksenberg A, Arons E, Nasser K et al. Severe obstructive sleep apnea: sleepy versus nonsleepy patients. Laryngoscope 2010; 120: 643-648

5 Bixler EO, Vgontzas AN, Lin HM et al. Excessive daytime sleepiness in a general population sample: the role of sleep apnea, age, obesity, diabetes, and depression. J Clin Endocrinol Metab 2005; 90: 4510-4515

6 Roure N, Gomez S, Mediano O et al. Daytime sleepiness and polysomnography in obstructive sleep apnea patients. Sleep Med 2008; 9: 727 731

7 Gottlieb DJ, Whitney CW, Bonekat WH et al. Relation of sleepiness to respiratory disturbance index: the Sleep Heart Health Study. Am J Respir Crit Care Med 1999; 159: 502-507 
8 Chen R, Xiong KP, Lian YX et al. Daytime sleepiness and its determining factors in Chinese obstructive sleep apnea patients. Sleep Breath published online $2010 \mathrm{Feb} 20$

9 Weeß HG, Sauter C, Geisler P et al. Vigilanz, Einschlafneigung, Daueraufmerksamkeit, Müdigkeit, Schläfrigkeit - Diagnostische Instrumentarien zur Messung müdigkeits- und schläfrigkeitsbezogener Prozesse und deren Gütekriterien. Somnologie 2000; 4: 20-38

10 Johns MW. A new method for measuring daytime sleepiness: The Epworth Sleepiness Scale. Sleep 1991; 14: 540-545

11 Johns MW. Daytime sleepiness, snoring, and obstructive sleep apnea: The Epworth Sleepiness Scale. Chest 1993; 103: 30 - 36

12 Schwarzenberger-Kesper F, Becker H, Penzel T et al. Die exzessive Einschlafneigung am Tage (EDS) beim Apnoe-Patienten: Diagostische Bedeutung und Objektivierung mittels Vigilanztest und synchroner EEGRegistrierung am Tage. Prax Klein Pneumol 1987; 41: 401 - 405

13 Aarand $D$, Bonnet $M$, Hurwitz $T$ et al. Review by the MSLT and MWT Task Force of the Standards of Practice Committee of the American Academy of Sleep Medicine. Sleep 2005; 28: 123-144

14 Conradt $R$, Hochban $W$, Heitmann J et al. Sleep fragmentation and daytime vigilance in patients with OSA treated by surgical maxillomandibular advancement compared to CPAP therapy. J Sleep Res 1998; 7: $217-223$

15 Cassel W, Ploch T, Becker $H$ et al. Risk of traffic accidents in patients with sleep-disordered breathing: reduction with nasal CPAP. Eur Respir J 1996; 9: 2606 - 2611

16 Sangal RB, Mitler MM, Sangal JM. Subjective sleepiness ratings (Epworth sleepiness scale) do not reflect the same parameter of sleepiness as objective sleepiness (maintenance of wakefulness test) in patients with narcolepsy. Clinical Neurophysiology 1999; 110: 2131 2135

17 Olson LG, Cole MF, Ambrogetti A. Correlations among Epworth Sleepiness Scale scores, multiple latency tests and psychological symptoms. J Sleep Res 1998; 7: 248-253

18 Benbadis SR, Mascha E, Perry MC et al. Association between the Epworth Sleepiness Scale and the multiple sleep latency test in a clinical population. Annals of Internal Medicine 1999; 130: 289-292

19 Mathis J, Hess CW. Sleepiness and vigilance test. SWISS MED WKLY 2009; 139: 214-219

20 Krystal AD, Edinger JE. Measuring sleep quality. Sleep Medicine ; 9(1): $10-17$

21 Levendowski DJ, Zack N, Rao S et al. Assessment of the test-retest reliability of laboratory polysomnography. Sleep Breath 2009; 13: 163 167

22 Young T, Palta M, Dempsey J et al. The occurrence of sleep-disordered breathing among middle-aged adults. N Engl J Med 1993; 328: 230 1235

23 Kapur VK, Baldwin CM, Resnick HE et al. Sleepiness in patients with moderate to severe sleep-disordered breathing. Sleep 2005; 28: 472 - 477

24 Dixon JB, Dixon ME, Anderson ML et al. Daytime sleepiness in the obese: Not as simple as obstructive sleep apnea. Obesity 2007; 15: 25042511

25 Mediano O, Barcelo A, de la Pena $M$ et al. Daytime sleepiness and polysomnographic variables in sleep apnoea patients. Eur Respir J 2007; 30: $110-113$

26 Tsaoussoglou M, Bixler EO, Calhoun S et al. Sleep-disordered breathing in obese children is associated with prevalent excessive daytime sleepiness, inflammation, and metabolic abnormalities. J Clin Endocrinol Metab 2010; 95: 43-150

27 Gozal D, Kheirandish-Gozal L. Obesity and excessive daytime sleepiness in prepubertal children with obstructive sleep apnea. Pediatrics 2010; 123: $13-18$

28 Vgontzas AN, Bixler EO, Tan TL et al. Obesity without sleep apnea is associated with daytime sleepiness. Arch Intern med 1998; 158: $1333-1337$

29 Vgontzas AN. Excessive daytime sleepiness in sleep apnea: It is not just apnea hyponea index. Sleep Medicine 2008; 9: 712 - 714

30 Vgontzas AN, Papanicolaou DA, Bixler EO et al. Sleep apnea and daytime sleepiness and fatigue: relation to visceral obesity, insulin resistance, and hypercytokinemia. Journal of Clinical Endocrinology and Metabolism 2000; 85: 1151 - 1158

31 Vgontzas AN, Bixler EO, Chrousos GP et al. Obesity and sleep disturbances: Meaningful sub-typing of obesity. Arch of Physiol and Biochemistry $2008 ; 114: 224-236$
32 De la Pena Bravo M, Serpero LD, Barcelo A et al. Inflammatory proteins in patients with obstructive sleep apnea with and without daytime sleepiness. Sleep Breath 2007; 11: 177 - 185

33 Marshall NS, Bames M, Travier $N$ et al. Continuous positive airway pressure reduces daytime sleepiness in mild to moderate obstructive sleep apnoea: a meta-analysis. Thorax 2006; 61: 430-434

34 Patel SR, White DP, Malhotra A et al. Continuous positive airway pressure therapy for treating sleepiness in a diverse population with obstructive sleep apnea. Arch Intern Med 2003; 163: 565 - 571

35 Black J. Sleepiness and residual sleepiness in adults with obstructive sleep apnea. Respiratory Physiology \& Neurobiology 2003; 136: 211 220

36 Sanders $M H$, Newmann AB, Haggerty $C L$ et al. Sleep and sleep-disordered breathing in adults with predominantly mild obstructive airway disease. Am J Respir Crit Care Med 2003; 167: 7-14 